Bull. Mater. Sci., Vol. 8, No. 2, May 1986, pp. 133-146. (C) Printed in India.

\title{
Computer modelling application in life prediction of high temperature components
}

\author{
V M RADHAKRISHNAN \\ Indian Institute of Technology, Madras 600036, India
}

\begin{abstract}
Defects introduced in pressure vessel components during fabrication processes act as potential sources for damage accumulation and subsequent catastrophic failure. Cracks nucleate at these stress risers and propagate aided by fatigue type of loading, corrosion and creep. Analysis of crack growth under conditions of 'time-dependent fatigue' is very important for the life prediction of pressure vessel components. In this paper the interaction of creep-hot corrosion and low cycle fatigue is analyzed based on the energy expended for the nucleation of damage at the advancing crack front. The total damage accumulation is divided into that due to (i) fatigue, (ii) corrosion and (iii) creep for modelling purpose. The analysis yields a relation in terms of $J$-integral which is applicable to both crack propagation and final failure. A corrosion-creep parameter $\left(F_{i}\right)$ has been introduced at the crack propagation stage and raw data from different sources have been analyzed for different types of loading and compared with the theoretical predictions. The total energy in tension which includes the tension going time, appears to be a good parameter for the prediction of time-dependent fatigue life.
\end{abstract}

Keywords. Life prediction; pressure vessel; high temperature components; fatigue life; computer model; crack growth model.

\section{Introduction}

Pressure vessels operating in the creep range include steam chests, loop pipes, HP and IP cylinders, turbine parts, valves and numerous items of pipe works. Many of these components will contain crack-like defects specifically at places where joints are made. As large components involve a high capital investment, maximum service life consistent with safety of operation has to be ensured. Failure of many high temperature pressure vessel components due to crack growth has been reported in the literature (Toft et al 1976). A typical example is the failure of a welded joint between IP steam chest and a loop pipe (figure 1a) in a $350 \mathrm{MW}$ unit plant in UK. The material of the steam chest was cast $1 \mathrm{Cr}-\mathrm{Mo}-\mathrm{V}$ steel and that of the loop pipe was $1 / 2 \mathrm{Cr}-1 / 2 \mathrm{Mo}-1 / 4 \mathrm{~V}$ steel. The steam was at 35 bar pressure and $560^{\circ} \mathrm{C}$. The welded joint between steam chest and the loop pipe failed explosively. Investigations revealed a sub-surface crack of $300 \mathrm{~mm}$ length in the heat-affected zone of the weld on the steam chest side. The crack was formed during the post-weld stress relief heat treatment and had grown in service. Another example is the failure of a superheater tube (figure $1 \mathrm{~b}$ ) of a $100 \mathrm{MW}$ plant. The material of the tube was $18 \mathrm{Cr}-12 \mathrm{Ni}-1 \mathrm{Nb}$ (AISI/347) steel and that of the spacers was $25 \mathrm{Cr}-20 \mathrm{Ni}$. The superheater tubes are kept in alignment and correctly spaced by the use of interlocking spacers which are welded to the tube, but free to move in the vertical plane to accommodate thermal expansion. Tube failures occurred after 12,000 hours of operation and examination revealed cracking at the fillet weld interface.

The significance of crack nucleation and its growth behaviour under dynamic 


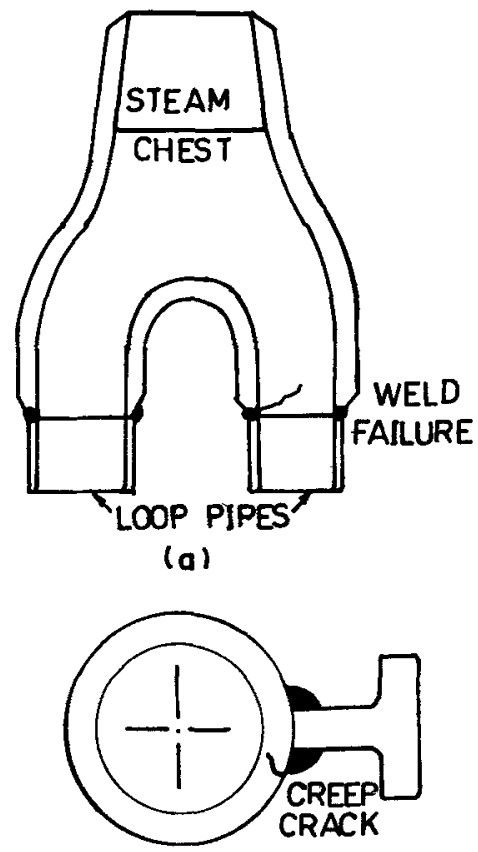

(b)

Figure 1. (a) IP steam chest and pipe joint. (b) superheater tube joint.

stresses where creep, corrosion and low cycle fatigue are the main contributing factors to damage processes has started receiving attention only recently. In this paper an analysis is presented to evaluate the time-dependent crack growth and its application in life prediction methods.

\section{Crack growth model}

In time-dependent fatigue, damage nucleation at the tip of the advancing crack could be in the form of micro-cracks or voids contributed by point and line defects. Both the strain energy at the crack tip $\delta W^{*}$ and the thermal energy will contribute to the damage nucleation ahead of the crack tip. The damage nucleated per cycle in a unit volume of the material ahead of the crack can be given as a rate equation in the form (Radhakrishnan 1982)

$$
\mathrm{d} D / \mathrm{d} N=A_{1} \exp \left(\frac{-Q+m^{\prime} \log \left(\delta W^{*} / W_{0}\right)}{k T}\right),
$$

where $Q$ is the activation energy and $A_{1}, m^{\prime}$ and $W_{0}$ are constants. A similar $\mathrm{d} a / \mathrm{d} N$ dependence ( $a=$ crack length) of the activation energy had been found to be valid in the case of high temperature fatigue of aluminium (Jeglic et al 1973). The above relation can be rewritten as

$$
\mathrm{d} D / \mathrm{d} N=A_{1}\left(\delta W^{*} / W_{0}\right)^{m} \exp (-Q / k T),
$$

where $m=m^{\prime} / k T$. 
The damage takes place over a small highly strained volume, represented by its linear dimension $\boldsymbol{R}_{p}$. In the case of elastic field this dimension can be identified as the width of the plastic zone given by

$$
R_{p}=\left(E / \sigma_{y s}^{2}\right) J_{e}
$$

where $E$ is the Young's modulus and $\sigma_{y s}$ the yield stress. $J_{e}$ is the $J$-integral corresponding to the stress field. In the case of high temperature low cycle fatigue, the width of the highly strained volume can be given by a similar type of relation in the form

$$
R_{p}=\left(E / \sigma_{y s}^{2}\right) \delta J
$$

The total damage in the highly strained zone joins the crack and the crack advances through a small distance $\mathrm{d} a$ at time $\mathrm{d} N$. Hence the crack growth rate can be given as

$$
R_{p}(\mathrm{~d} D / \mathrm{d} N)=A_{1}\left(E / \sigma_{\mathrm{ys}}^{2}\right)(\delta J)\left(\delta W^{*} / W_{0}\right)^{m} \exp (-Q / k T) .
$$

The energy density $\delta W^{*}$ at the crack front can be related to the applied energy density $\delta W$ and the crack length $a$ by (Radhakrishnan 1980a)

$$
\delta W^{*}=(4 / r)(\delta W \cdot a),
$$

where $r$ is the crack tip radius. It can also be shown that the $J$-integral range $\delta J$ is given by a function of the type (Kaisand and Mowbray 1979)

$$
\delta J=\text { constant }(\delta W \cdot a),
$$

where $\delta W$ is the work done during the tensile portion of loading. With (6) and (7) we can write the crack propagation rate as

$$
\mathrm{d} a / \mathrm{d} N=A_{2}(\delta W \cdot a)^{m+1}=A_{3}(\delta J)^{m+1},
$$

where $A_{2}$ and $A_{3}$ are constants which include all the other constants.

Under certain conditions of loading, as in vacuum, some of these damages nucleated at the crack front may get healed during compressive loading. Under normal conditions of testing with ambient air, such healing may not be possible. In addition, with hold time or with low frequency, the crack front will be subjected to environmental attack. Hofflener and Speidel (1979) reported that the crack propagation rate is twice higher in air than in vacuum for IN $738 \mathrm{LC}$ and IN 939 alloys at $850^{\circ} \mathrm{C}$. In addition to the fatigue and corrosion effects, if the tensile-going frequency is sufficiently low, then creep effect will also come into the picture which will generate some more defects at the crack front thereby increasing the crack growth rate. The basic model of the contributions to the crack growth due to the three factors, namely, fatigue, corrosion and creep is schematically shown in figure 2 . In these components, $(\delta a)_{f}$ may be considered to be due to PP type of loading (only due to fatigue). The corrosion component $(\delta a)_{\text {cor }}$ is added when the frequency is low or when there is a hold period. Oxide layers will form and if the scale that forms spalls off, then further corrosion may take place. Or else, the corrosion effect will slow down and hold times beyond a certain value will not add much to the damage accumulation. The corrosion effect may be taken as a log function (or any other suitable function) of the time of exposure of the newly formed surface-in this analysis the tensile going time-and can be given by

$$
\delta a_{\text {cor }}=A_{\text {cor }} \log \left(t / t_{0}\right) \text { func }(\delta J),
$$

where $A_{\text {cor }}$ and $t_{0}$ are constants. 


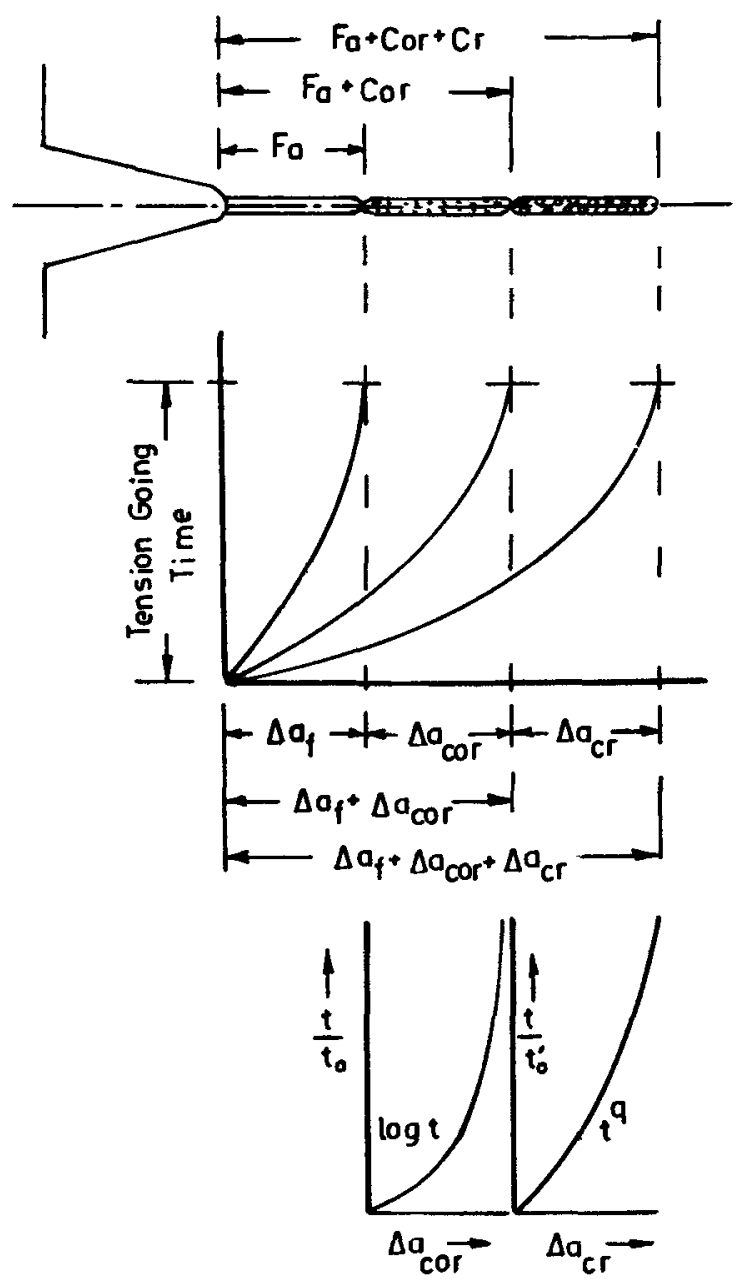

Figure 2. Crack growth model.

When the frequency is very low and the stress generated due to the applied strain range is sufficiently high at the operating temperature to cause creep deformation, then creep damage will also occur at the crack tip during the tensile portion of the cycle. The essential condition is that the creep rate due to that particular stress level must be higher than the imposed strain rate. The creep damage under such a condition can be assumed to be a function of the creep strain. Since during each cycle load is applied afresh, the creep strain will be in the transient stage and so it will follow generally the one third law. So the contribution due to creep for the crack growth, i.e., $\delta a_{\text {creep }}$ can be taken as a power function of the tensile going time and can be written as

$$
\delta a_{\text {creep }}=A_{\text {cr }}\left(t / t_{0}^{\prime}\right)^{q} \text { func }(\delta J),
$$

where the value of the exponent $q=0 \cdot 33$. These growth phenomena are assumed to take place during the tensile going frequency and hence this treatment can be applied to unbalanced hysteresis loops and tensile hold periods. 
Now combining the effects of corrosion and creep, the crack growth during each cycle can be given as

$$
\delta a=\delta a_{f}+\delta a_{\text {cor }}+\delta a_{\mathrm{cr}}
$$

which yields the generalized relation in the form (equation (8))

$$
\mathrm{d} a / \mathrm{d} N=A_{f}\left(1+\alpha \log \left(t / t_{0}\right)+\beta\left(t / t_{0}^{\prime}\right)^{q}\right)(\delta J)^{m+1},
$$

where $\alpha=A_{\text {cor }} / A_{f}$ and $\beta=A_{\mathrm{cr}} / A_{f} . t_{0}$ and $t_{0}^{\prime}$ are the tensile-going times (the corresponding frequencies being $v_{0}$ and $v_{0}^{\prime}$ ) beyond which corrosion and creep will be active respectively.

The above relation is also applicable when there is a hold period in the stress-strain cycling. When there is a stress hold, the creep effect will come into the picture and the term $\beta\left(t / t_{0}^{\prime}\right)^{q}$ will have to be taken in the equation. But when there is a strain hold, then relaxation of stress will take place. Some of the cavities created during the peak stress may collapse. So the effect of stress relaxation will be similar to corrosion and the contribution due to the damage accumulation can be similar to that of corrosion in the form

$$
(\delta a)_{\text {relaxation }}=A_{r} \log \left(t / t_{0}\right) \text { func }(\delta J)
$$

\section{Data analysis}

Expressing the $J$ range $(\delta J)$ in terms of LEFM parameter $\delta K$ in the form $\delta J=\delta K^{2} / E$, relation (12) can be written as

$$
\mathrm{d} a / \mathrm{d} N=A_{f}\left(F_{i}\right)(\delta K)^{2(m+1)},
$$

where $A_{f}$ is a constant for a given material and temperature. $F_{i}$ is the interaction parameter and is equal to $1+\alpha \log \left(t / t_{0}\right)+\beta\left(t / t_{0}^{\prime}\right)^{q}$ or $\alpha^{\prime} \log \left(t / t_{0}\right)$. Equation (14) is similar to the Paris-Erdogan law given as

$$
\mathrm{d} a / \mathrm{d} N=C(\delta K)^{n} .
$$

Crack growth data (James 1972) obtained on a 304 type stainless steel tested at $538^{\circ} \mathrm{C}$ over a wide frequency range of $6.67 \mathrm{~Hz}$ to $0.0014 \mathrm{~Hz}$ were analyzed based on (14) and the relation between $\mathrm{d} a / \mathrm{d} N$ and $\delta K$ is shown in figure 3 , with the values of the constant $A_{f}=1.7 \times 10^{-8} \mathrm{~mm} /(\mathrm{MPa} \sqrt{\mathrm{m}})^{3}, \alpha=0.2$ and $\beta=0.75$. As the frequency decreases there is a decrease in the value of the exponent $(m+1)$. It has been shown (Radhakrishnan 1980b) that in the Paris equation describing the state II crack growth, the constant $C$ and the exponent $n$ are inter-related. As $C$ increases the value of $n$ decreases. In a similar manner, in this case also, as frequency is lowered, the interaction factor $\left(F_{i}\right)$ in (14) will increase with a consequent decrease in the value of the exponent $(m+1)$.

Equation (14) can be rearranged and integrated with crack length $a$ from initiation $a_{i}$ to critical length $a_{f}$ and the number of cycles $N$ from nucleation time $N_{i}$ to final fracture $N_{f}$. Assuming the nucleation of the crack to start within a first few cycles and $a_{i} \ll a_{f}$, it can be shown

$$
\left(F_{i}\right)\left(\delta W_{t}\right)^{m+1} N_{\mathrm{fi}}=\text { constant }
$$




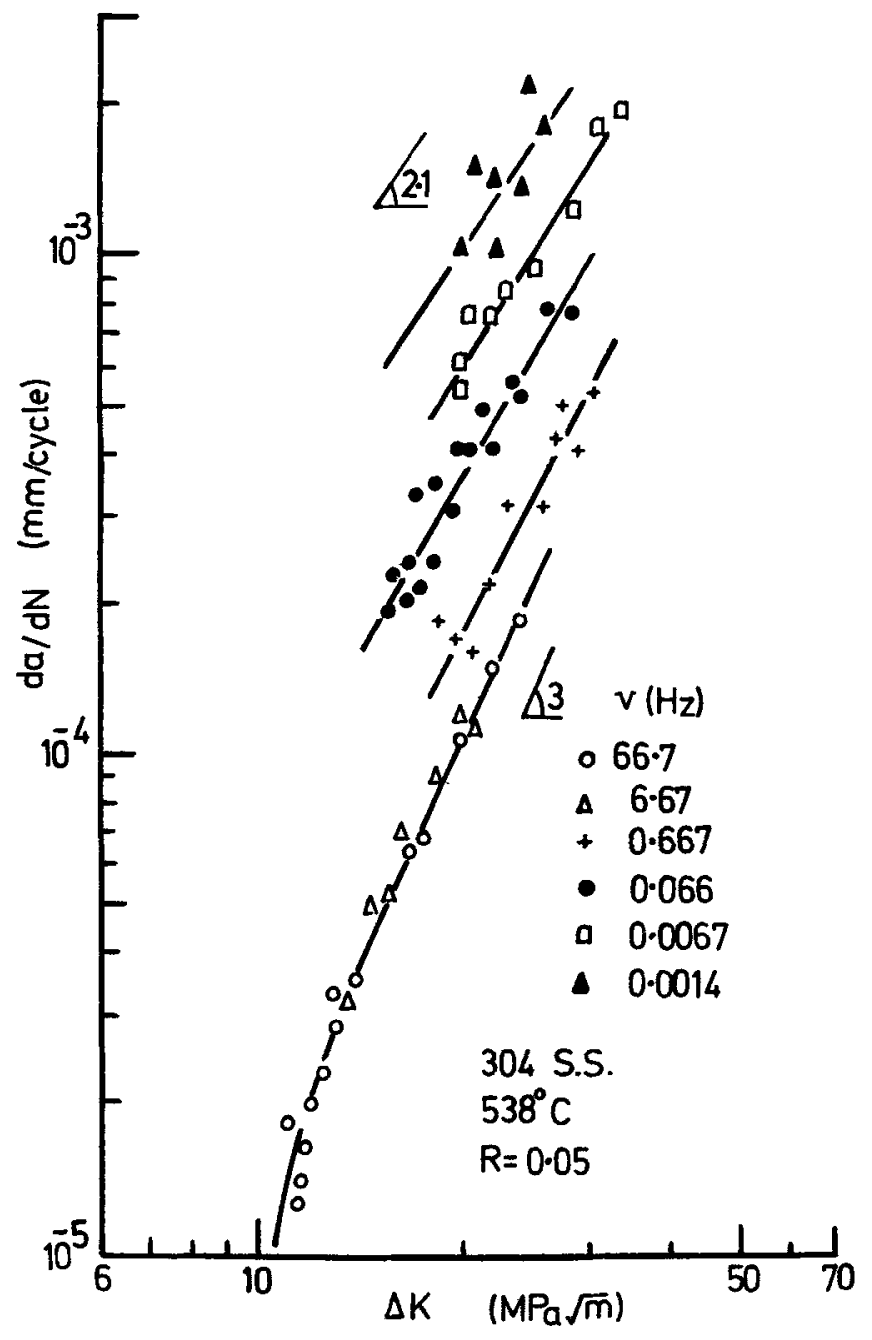

Figure 3. Relation between $\mathrm{d} a / \mathrm{d} N$ and $\delta K$.

where $\delta W_{\mathrm{t}}$ is the tensile energy input per cycle per unit volume. $N_{\mathrm{fi}}$ is the number of cycles to failure under interaction conditions. If $\left(F_{i}\right)=1$, the $N_{\mathrm{fi}}=N_{f}$. If the cyclic stress-strain relation is known, $\delta W_{t}$ can be expressed in terms of $\delta \varepsilon$ or $\delta \sigma$. Thus the usual endurance life relation can be established. It can be seen that decreasing the frequency will shift the $\delta \varepsilon-N_{\mathrm{ff}}$ relation downwards on the $\log -\log$ plot. The slope of these lines will be proportional to $1 /(m+1)$ and since the value of $(m+1)$ decreases with decreasing frequency or increasing tensile hold period, the slope of the lines $\delta \varepsilon-N_{\mathrm{ff}}$ on the $\log -\log$ plot will increase, thereby showing a rotational effect in addition to a downward shift as the frequency is reduced.

The energy associated with the tensile portion of the cycle depends on the wave shape-typical examples of which are shown in figure 4. In the case of a balanced hysteresis loop with no creep effect, the energy associated with the $J$ integral will be $\delta W_{e}$ 

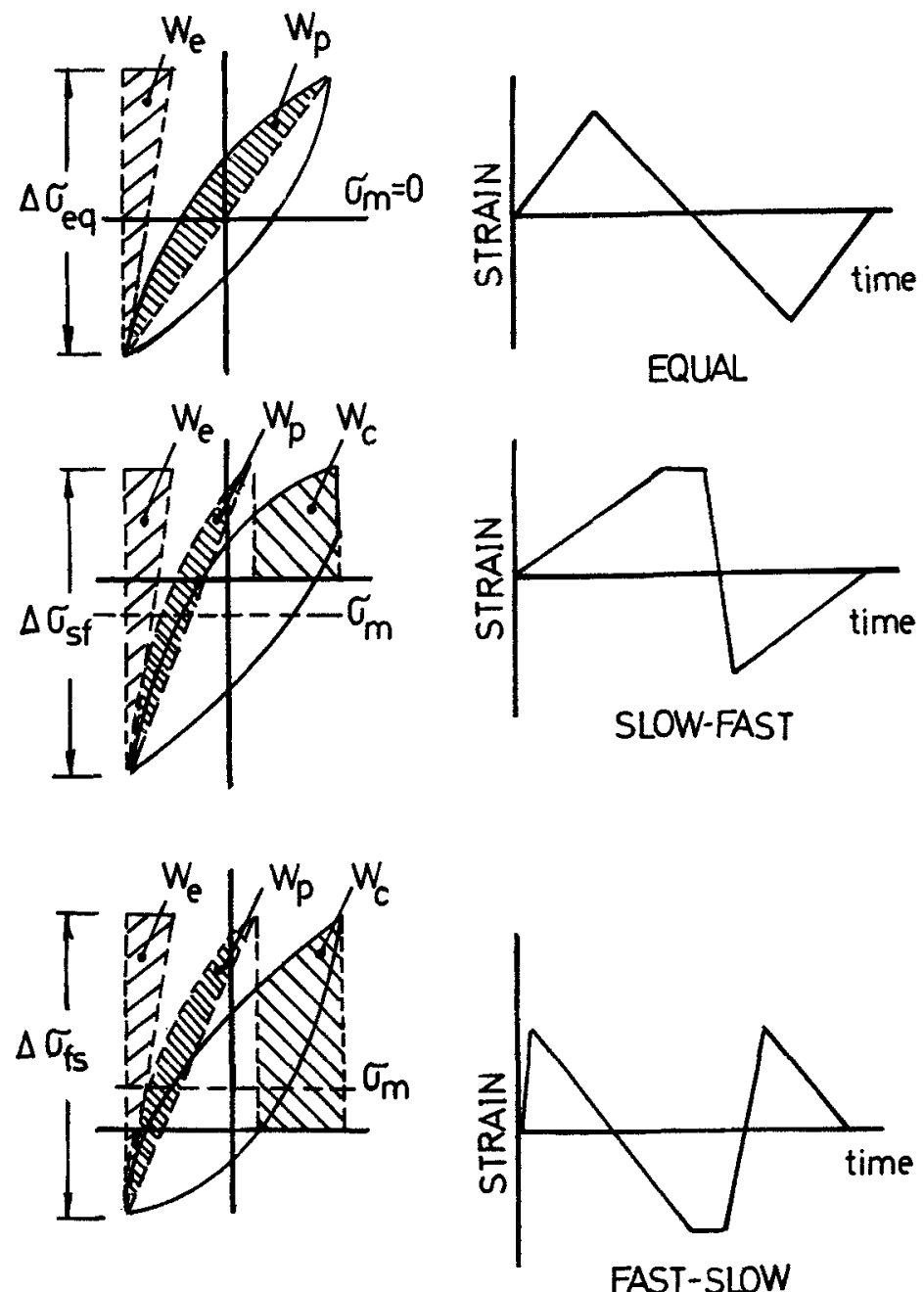

Figure 4. Energy separation method.

and $\delta W_{p}$, the elastic and plastic energy corresponding to $\delta J_{e}$ and $\delta J_{p}$. This energy term will be

$$
\begin{aligned}
\delta W_{t} & =\delta W_{e}+\delta W_{p} \\
& =\frac{\delta \sigma}{2} \delta \varepsilon_{\mathrm{el}}+\frac{\left(1-n^{\prime}\right)}{\left(1+n^{\prime}\right)} \frac{\delta \sigma}{2} \delta \varepsilon_{\mathrm{pl}}
\end{aligned}
$$

The second term corresponding to the plastic region depends on the cyclic strain hardening exponent $n^{\prime}$ under the PP type of loading. At high temperatures where the hardening of the material drops, the value of the exponent $n^{\prime}$ will be small $(=0 \cdot 1$ to $0 \cdot 15)$ and in such a case relation (16) can be written as

$$
\left(F_{i}\right)\left(\sigma_{t} \delta \varepsilon_{t}\right)^{m+1} N_{f}=\text { constant }
$$

Raw data from Taraneshi and McEvily (1981) have been analyed and typical relations 
between the tensile energy $\delta W_{t}$ and the cycles to failure $N_{f}$ for 800 and $800 \mathrm{H}$ nickel alloys are shown in figure 5 . Here the cycling was rather continuous and the influence of creep could be negligible.

Even when the stress or strain is held constant for a duration either in tension or in compression, if the temperature is not high enough to cause creep damage or stress corrosion cracking in the small interval of time, the interaction parameter $\left(F_{i}\right)$ in (16) will almost be equal to unity and the relation between $\delta W_{t}$ and $N_{f}$ on $\log -\log$ scale will be a straight line for continuous cycles, tension hold ( $S F)$, compression hold $(F S)$ and tension-compression hold (SS). Such a typical correlation at comparatively low temperature where creep damage effect may be negligible, is shown in figure 6 for Cr-Mo steel.

The need to consider the unbalancing in hysteresis loop comes in at temperatures where corrosion and creep effects will be more pronounced. In the model presented here, the entire tensile energy term is divided into three components, $W_{e}, W_{p}$ and $W_{\text {cr }}$ - the last term being due to tensile stress $\sigma_{t}$ and the displacement $\varepsilon_{\text {creep }}$. So $\delta W_{t}$ is written as

$$
\delta W_{t}=\frac{\delta \sigma}{2} \delta \varepsilon_{e}+\frac{\left(1-n^{\prime}\right)}{\left(1+n^{\prime}\right)} \frac{\delta \sigma}{2} \delta \varepsilon_{p}+\sigma_{\imath} \delta \varepsilon_{\mathrm{c}}
$$

where $\delta \varepsilon_{\mathrm{el}}+\delta \varepsilon_{\mathrm{pl}}+\delta \varepsilon_{\mathrm{cr}}=\delta \varepsilon_{\text {total }}$. The third term need not necessarily be due to creep under constant stress hold. When the frequency is very low and the stress developed is high enough to cause a creep rate which is higher than the imposed strain rate, the effect of creep will come in. Since the tensile portion of the energy is considered in the analysis, the type of tests PP(FF) and PC(FS) can be considered to be similar and that of CC(SS) and $C P(S F)$ will be similar excepting for the mean stress effect. Figure 7 shows the relation between the tensile energy and the number of cycles to failure under interaction conditions for a turbine disc alloy tested at $750^{\circ} \mathrm{C}$. It can be seen that PP and PC types

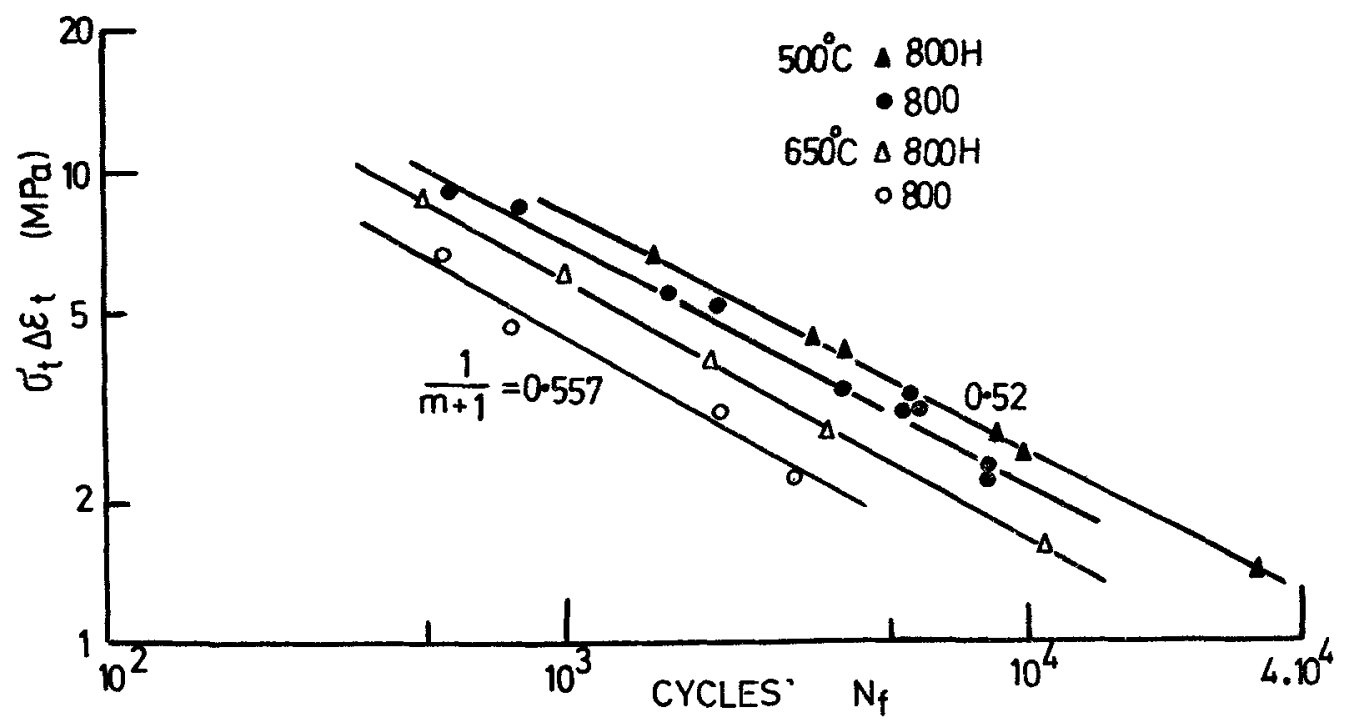

Figure 5. Relation between tensile energy density and cycles to failure. 


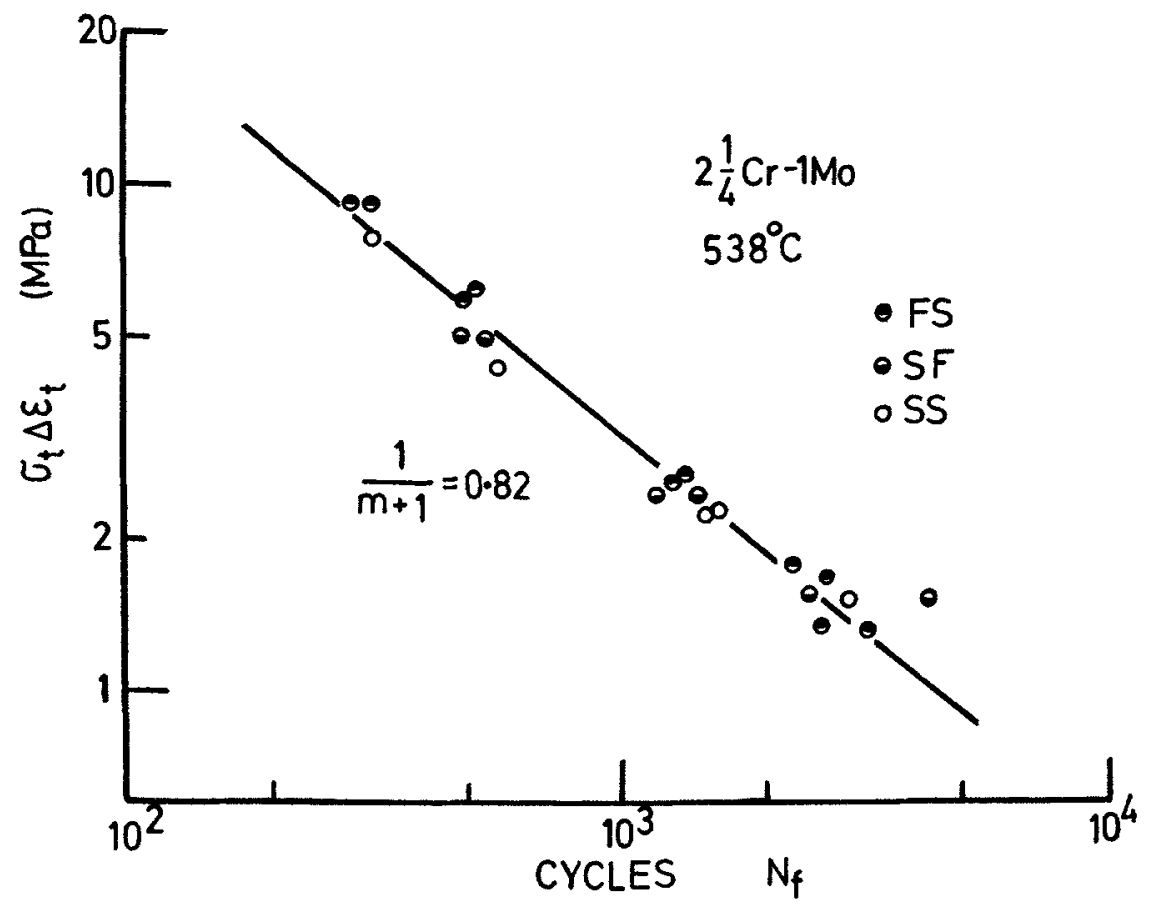

Figure 6. Relation between $\delta W_{t}$ and $N_{f}$.

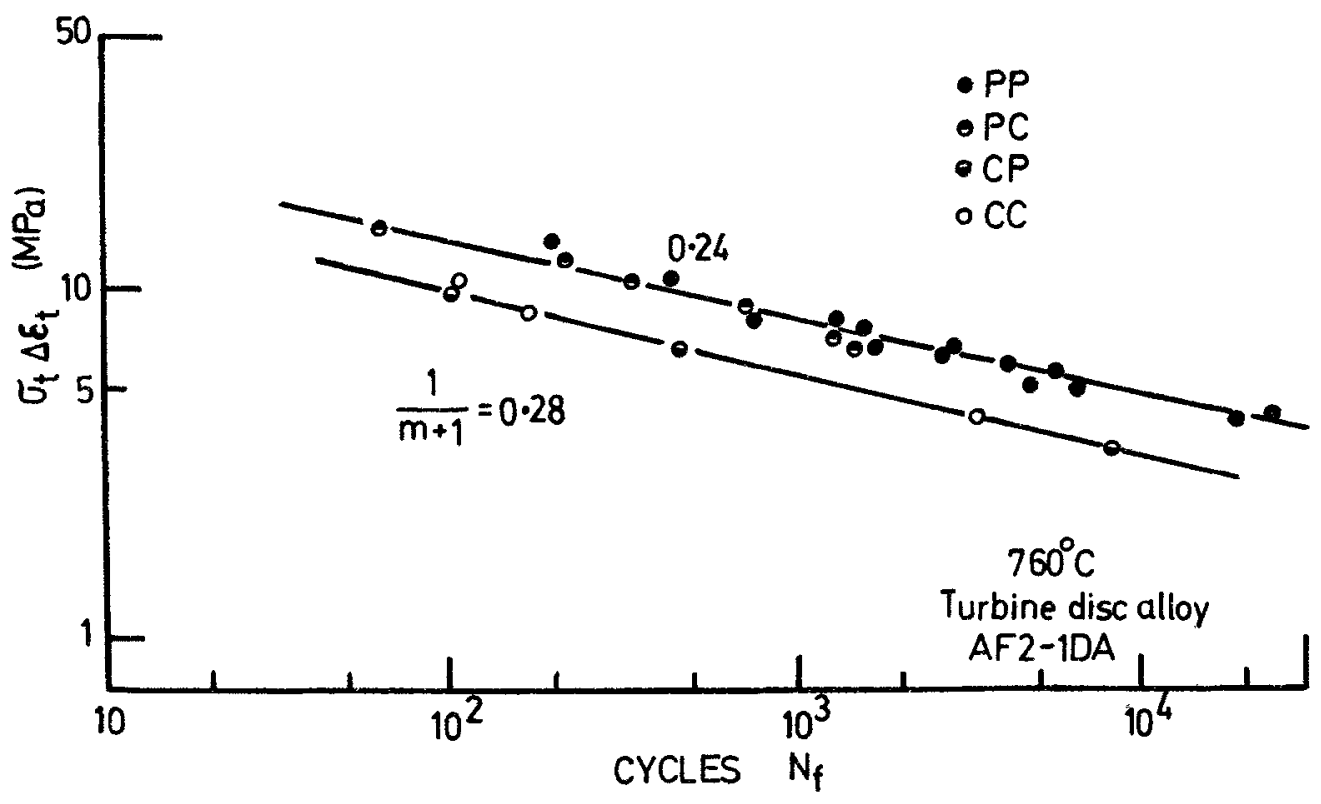

Figure 7. Relation between $\delta W_{t}$ and $N_{f}$. 
of loading data fall on one line and that of CP and CC on the other. The interaction factor $\left(F_{i}\right)$ was not taken into consideration. This has resulted in two separate lines for these two types of loading schemes. Figure 8 shows the relation between tensile energy and the number of cycles to failure under different loading frequencies and tensile hold times for type 304 stainless steel. Tensile strain hold times up to 600 minutes were employed. It can be seen from the results that when there is no hold time and the test is of PP type, (curve corresponding to $4 \mathrm{E}-03$ ) the slope of the line is $0.5(=1 /(1+m)$ ). As the hold time is introduced or as the frequency is reduced the slope increases to 0.525 and 0.625 as indicated in the figure and the interaction factor $\left(F_{i}\right)$ also increases, because of the corrosion and creep effects.

In (18) which can be written in the form

$$
N_{\mathrm{fi}}=\text { const. } \frac{\left(\delta W_{t}\right)^{-(m+1)}}{\left(F_{i}\right)},
$$

the exponent $(m+1)$ is dependent on the interaction factor $\left(F_{i}\right)$, as was discussed earlier, and it decreases as the value of $\left(F_{i}\right)$ increases. This relation can be given in the form

$$
(m+1)=A-\gamma \log \left(F_{i}\right)
$$

where $A=m+1$ when there is no interaction i.e., $\left(F_{i}\right)=1 . \gamma$ is a constant. As discussed in figure 8, the value of the constant $\gamma$ is taken as 1.2 for 304 stainless steel at $593^{\circ} \mathrm{C}$.

In many situations it is advantageous to represent the failure cycles $N_{\mathrm{fi}}$ as a function of tensile cycle time or tensile hold time. Figure 9 shows the relation between $N_{\mathrm{fi}}$ and the cycle time for cast 316 stainless steel and 304 stainless steel. It can be seen that for lower values of the strain range $\left(\delta \varepsilon_{t}=0.5 \%\right)$ the reduction in interaction cycle for larger

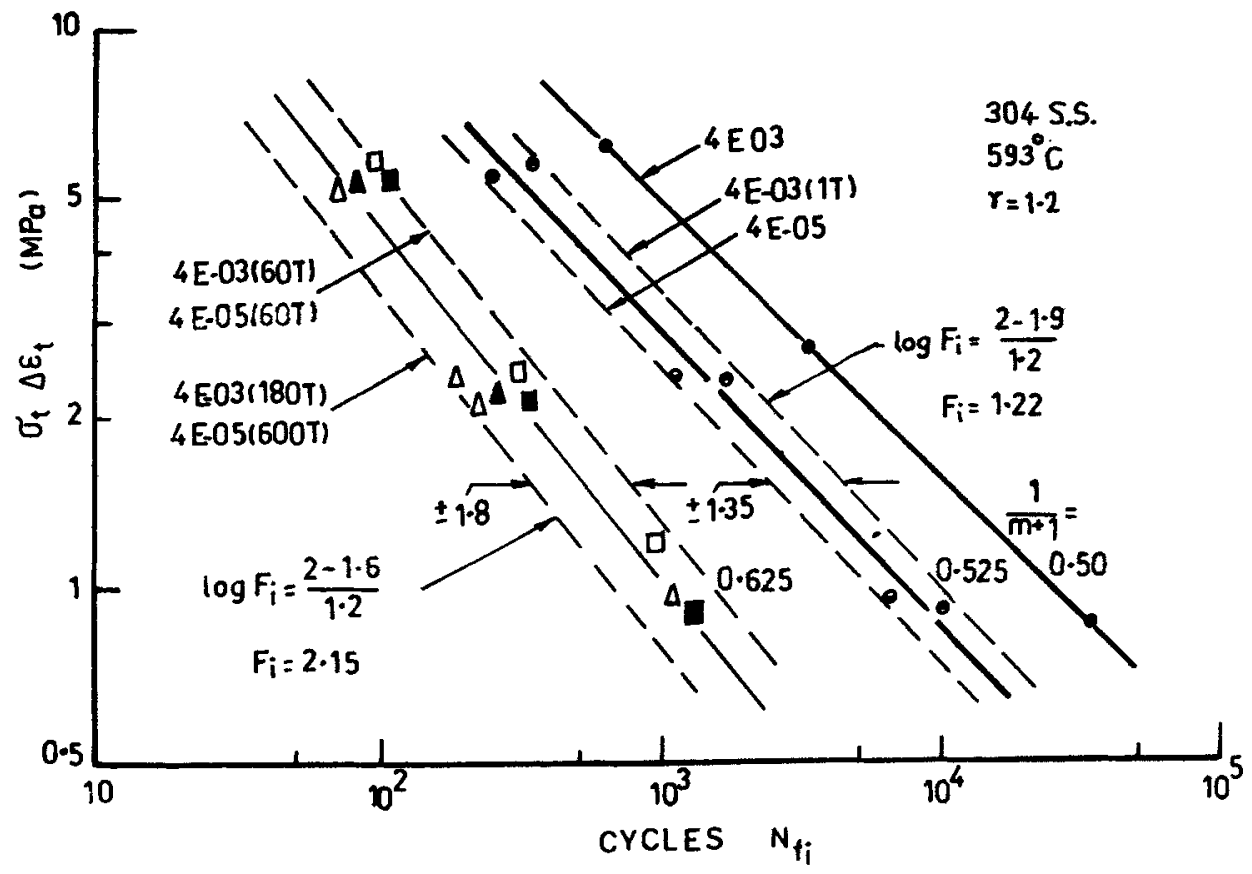

Figure 8. Relation between $\delta W_{t}$ and $N_{f}$. 

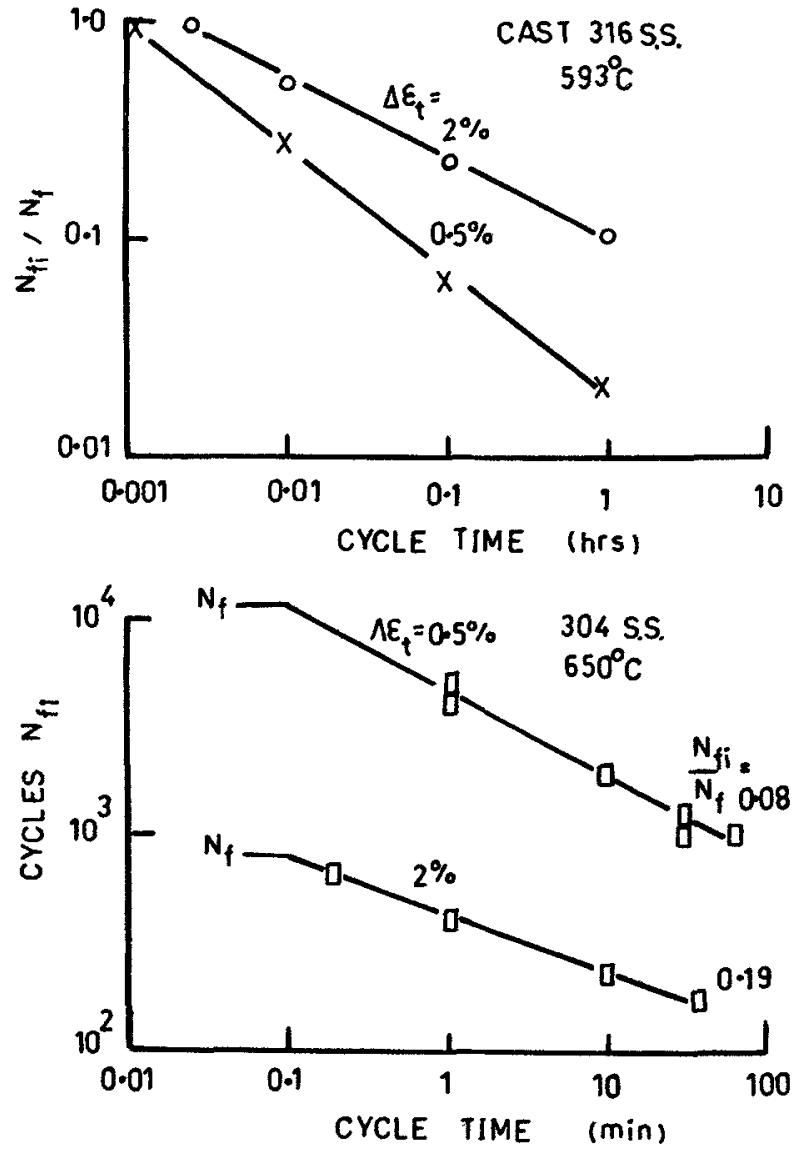

Figure 9. Relation between $N_{\mathrm{fi}}$ and cycle time.

cycle time is much higher than for higher strain ranges $\left(\delta \varepsilon_{t}=2 \%\right.$ ). Equation (20) is able to explain such a behaviour that has been observed in many other cases also. Assuming a simple relation between stress and strain in the form $\delta \sigma=\sigma_{0}(\delta \varepsilon)^{n^{\prime}}$ so that $\delta W_{t} \propto(\delta \varepsilon)^{n^{\prime}+1}$ we get the relation $(20)$ as

$$
(\delta \varepsilon)^{\left(n^{\prime}+1\right)(m+1)} \quad N_{f}=\text { constant, }
$$

for PP type of loading and

$$
\left(F_{i}\right)(\delta \varepsilon)^{\left(n^{\prime}+1\right)\left(A-\gamma \log F_{i}\right)} \quad N_{\mathrm{fi}}=\text { constant, }
$$

for interaction conditions. The fatigue life under interaction condition $N_{\mathrm{fi}}$ can thus be written as a function of the normal fatigue life $N_{f}$ as

$$
\frac{N_{\mathrm{fi}}}{N_{f}}=\frac{(\delta \varepsilon)^{\left(n^{\prime}+1\right) \gamma \log F_{i}}}{\left(F_{i}\right)}
$$

The above relation shows that $N_{\mathrm{fi}} / N_{f}$ is dependent on the applied strain range-the higher the strain range, the larger is the life ratio. This is what has been observed in the data presented in figure 9 (Wareing 1981). 
Equation (23) forms the basis for the construction of the interaction diagram i.e., the relation between the fatigue life under interaction conditions and the tensile going time which can include the stress or strain hold periods. In the case of strain-hold, the interaction factor $\left(F_{i}\right)$ becomes

$$
\begin{aligned}
F_{i} & =1+\alpha \log \left(t / t_{0}\right)+\alpha^{\prime} \log \left(t / t_{0}\right) \\
& =1+\left(\alpha+\alpha^{\prime}\right) \log \left(t / t_{0}\right)
\end{aligned}
$$

In the case of stress hold, it is given as

$$
F_{i}=1+\alpha \log \left(t / t_{0}\right)+\beta\left(t / t_{0}^{\prime}\right)^{q} .
$$

Figure 10 shows the interaction diagram for 304 type stainless steel. The full line is obtained with the interaction factor $F_{i}$ as given by (24) as this is a case of strain hold and stress relaxation can be expected to occur during the hold period. When the hold is very small, it is the same as normal fatigue without any interaction effect. As the hold time is increased, corrosion comes into the picture. Still further increase in hold time gives rise to corrosion and relaxation to occur. The value of $\left(\alpha+\alpha^{\prime}\right)$ is taken as $0 \cdot 2$. Here again it can be seen that the reduction in fatigue life is much more pronounced when the strain range is small.

Figures 11 and 12 show the relation between $N_{\mathrm{fi}}$ and the tensile hold period under both stress and strain hold conditions for 304 type strainless steel at $650^{\circ} \mathrm{C}$ and IN597 alloy at $850^{\circ} \mathrm{C}$. It can be seen that with stress-hold the relation between $N_{\mathrm{fi}}$ and time on the log-log plot becomes more or less a straight line with a negative slope approximately equal to one at higher hold periods. This means that the life becomes more timedependent than cycle dependent as the stress hold period is increased. At high tensile

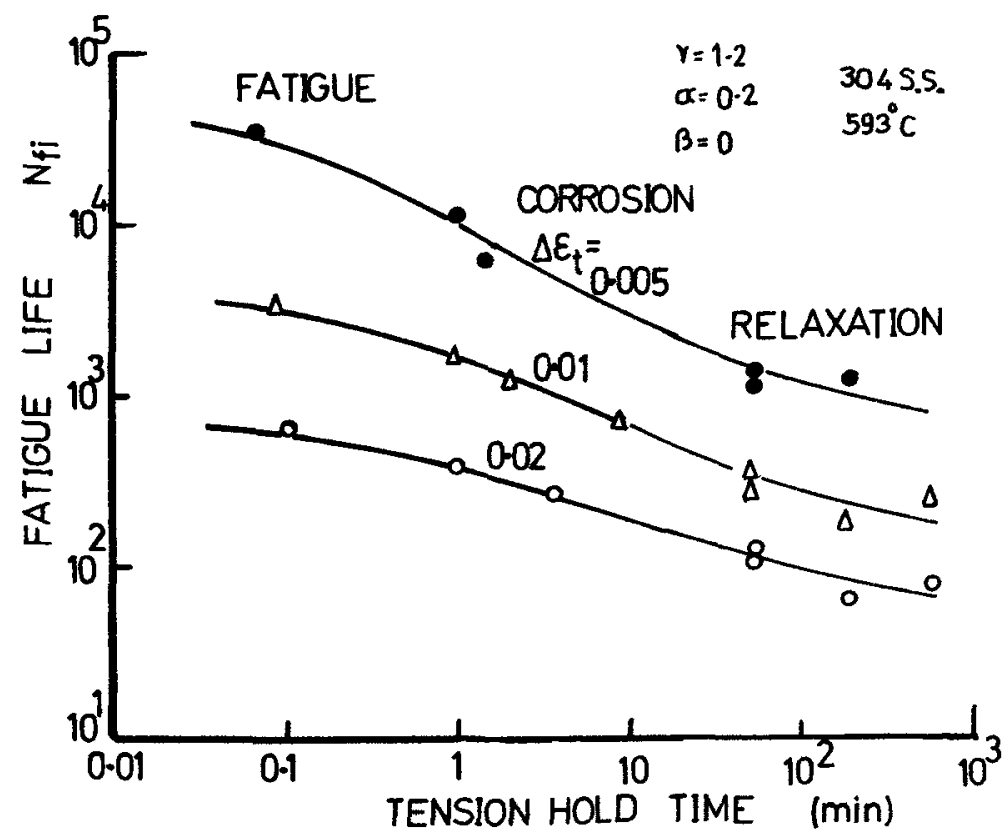

Figure 10. Interaction diagram for 304 stainless steel at $593^{\circ} \mathrm{C}$. 


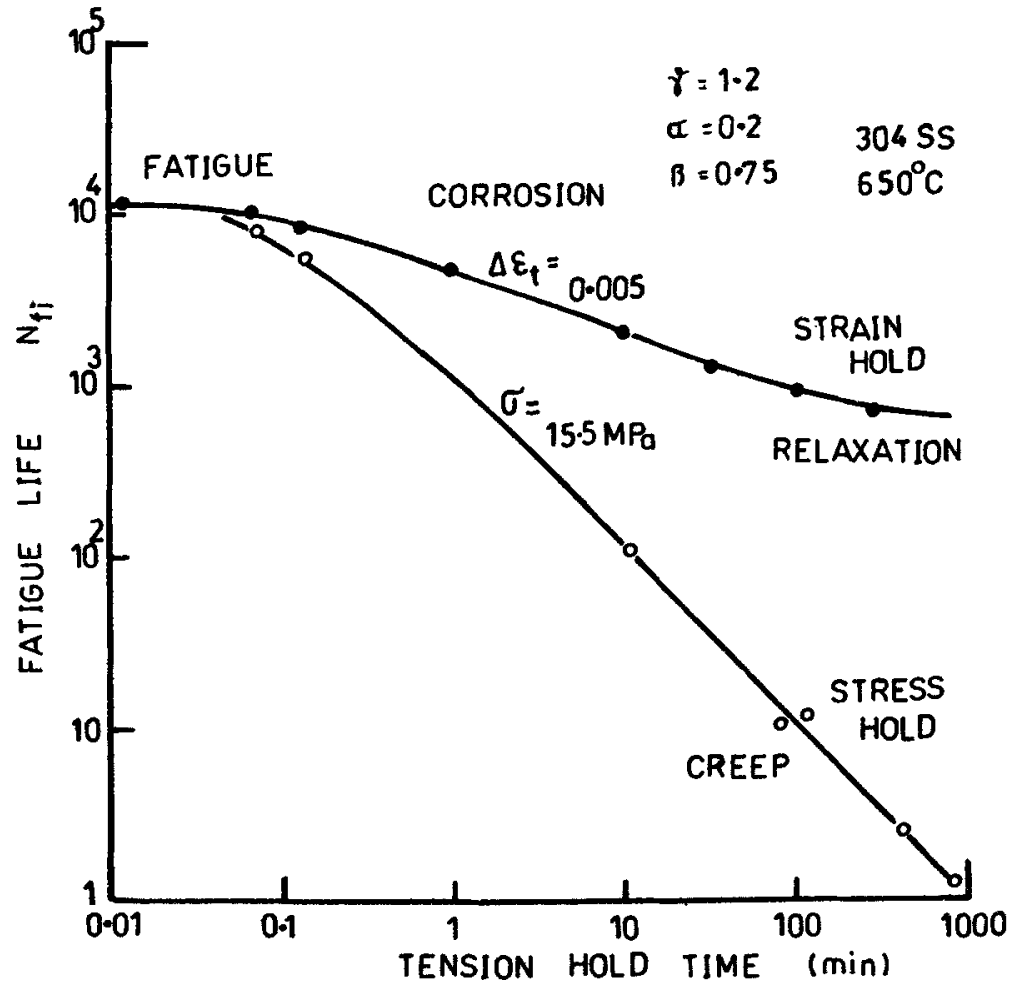

Figure 11. Interaction diagram for 304 stainless steel at $650^{\circ} \mathrm{C}$.

hold periods creep plays a dominant role under stress hold conditions. But under strain-hold conditions, a saturation level is reached and the fatigue life appears to be independent of the hold period beyond a certain stage, as relaxation governs the crack tip conditions. The same trend has been observed in all the cases (Radhakrishnan 1983).

The variables involved in the analysis of life prediction of components at high temperature are quite large. Various phenomena like hot corrosion, low cycle fatigue, creep, relaxation and thermal fatigue take place either individually or two or more combinations of them. In addition to these mechanical type of considerations, metallurgical factors such as chemical attack on grain boundaries and general structural degradation are also to be considered. In the model proposed, only the mechanical aspects are considered. This can be improved and extended to metallurgical variables also as more data are analysed.

\section{Concluding remarks}

In trying to model the damage accumulation and thereby predict the life of the component it is very important to know all the parameters that affect the damage process and their relative weightage. The logic and the governing equations are to be properly evaluated to build the sequence block diagram. 


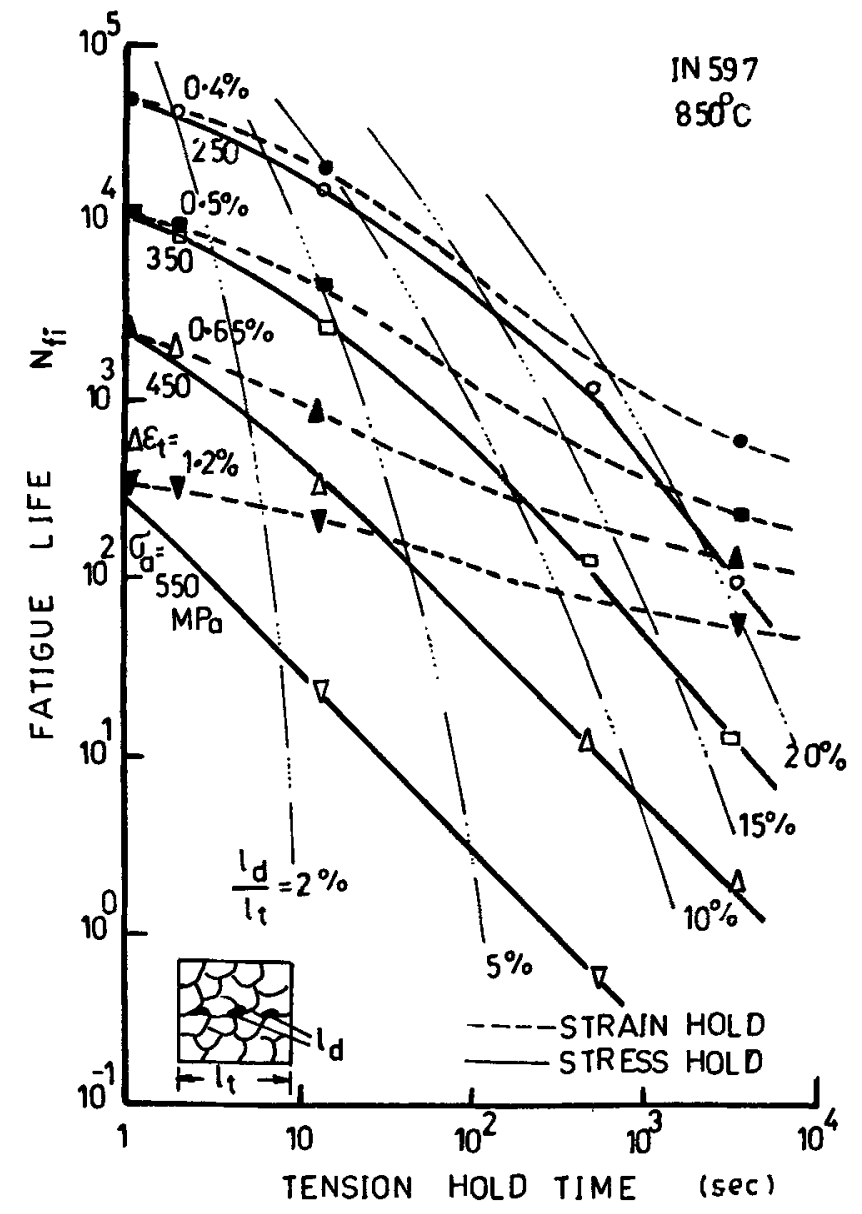

Figure 12. Interaction diagram for IN 597 alloy at $850^{\circ} \mathrm{C}$.

\section{References}

Hofflener W and Speidel M O 1979 Proc. Int. Conf. on High Temperature Alloys in Aggressive Environments, Petten, The Netherlands, 993

James L A 1972 American Society for Testing Materials Special Technical Report 513218

Jeglic F, Niesen P and Burns D J 1973 ASTM STP 520139

Kaisand L R and Mowbray D F 1979 J. Test. Eval. 7270

Radhakrishnan V M 1980a Fatigue Engg. Mater. Struct. 375

Radhakrishnan V M 1980b Engg. Fract. Mech. 13129

Radhakrishnan V M 1982 Damage accumulation and fracture life in low cycle high temperature fatigue, ASTM STP 770135

Radhakrishnan V M 1983 Interaction diagram for time-dependent fatigue 1983 Trans. A.S.M.E. JEMT 105 273

Taraneshi H and McEvily A J 1981 Proc. ICF-5 Cannes, 1981

Toft L H, Yeldham D E and Plastow B A 1976 Proc. Conf. Failure of Components operating in Creep Range I. Mech. E., London (1976) p. 107

Wareing 1981 J. Fatigue Engg. Mater. Struct. 4131 